\title{
Shear-Induced Stress Relaxation in a Two-Dimensional Wet Foam
}

\author{
John Lauridsen \\ Michael Twardos \\ Michael Dennin \\ Department of Physics and Astronomy \\ and Institute for Interfacial and Surface Science \\ University of California at Irvine \\ Irvine, CA 92697-4575.
}

(November 16, 2018)

We report on experimental measurements of the flow behavior of a wet, two-dimensional foam under conditions of slow, steady shear. The initial response of the foam is elastic. Above the yield strain, the foam begins to flow. The flow consists of irregular intervals of elastic stretch followed by sudden reductions of the stress, i.e. stress drops. We report on the distribution of the stress drops as a function of the applied shear rate. We also comment on our results in the context of various two-dimensional models of foams.

83.80.Iz,82.70.-y

Foams are ubiquitous in nature [1] and part of a larger class of materials which exhibit a type of behavior that has come to be called "jamming" 2, Loosely speaking, a "jammed" material is one that is unable to flow, typically due to the packing of the constituent particles. For the case of foams, which are composed of gas bubbles separated by fluid walls, the jamming is a consequence of the topological constraints that develop as the bubbles press against each other. The existence of a jammed state contributes to the complex flow behavior of foams. This flow behavior is one reason that foams are so interesting from both a fundamental and applied point of view [1]. For sufficiently small strains or stresses, a foam acts as an elastic solid (the jammed state). However, when the strain (or the stress) exceeds a critical value, known as the yield strain, a foam begins to flow. Of particular interest is the flow behavior of foams for small rates of strain. This flow occurs through "avalanches", or sudden nonlinear rearrangements of the bubbles in the foam that correspond to a decrease in the average energy, or stress, of the foam. In this paper, we will refer to these events as "stress drops". This type of behavior is common to many jammed systems where flow occurs in an irregular or stick-slip type manner when the system is near the transition to jamming [5]. One of the outstanding questions for flowing foams is the nature of the distribution of the sizes of the stress drops during this irregular flow.

One reason that this remains an open issue is that predictions for the distribution of stress drop sizes are somewhat model dependent. Various models of twodimensional foams have been used to study the connection between topological rearrangements and flow properties as a function of applied rate of strain [6 13]. All of these simulations predict the same qualitative behavior for the macroscopic flow for sufficiently small rates of strain that was described above: elastic behavior below a yield strain and nonlinear bubble rearrangements above the yield strain. However, the quantitative predictions for the size distribution and frequency of these events are model dependent. The dependence is predominately due to different assumptions concerning the source of dissipation in foams and treating foams with different degrees of "dryness". Distinguishing between these different models, and their underlying assumptions, experimentally is an important step in our understanding of foam rheology.

Two-dimensional foams may be characterized by the area fraction of gas, $\phi$. For $\phi<0.84$, foams "melt" into a froth of exclusively circular bubbles. Near this transition, where bubbles are predominately circular, a foam is said to be "wet". In the limit $\phi$ approaches 1 , the bubbles become polygonal with infinitely thin walls, and a foam is said to be "dry". The simulations studied by Kawasaki, et al. (6) are based on the vertex models of foams. This model assumes infinitely thin walls and only treats the dynamics of the vertices. As such, it is applicable to dry foams and unlikely to apply to the wetfoam system studied in this work. The simulations of Weaire, et al. 11, 12] focus on the behavior of foams under quasi-static, extensional flow. These are applicable to wet foams and, because they are quasistatic, contain no dissipation. These simulations measure the number of T1 events. A T1 event is a topological change that involves the switching of neighbors between bubbles. Reference 12] reports events that include large numbers of T1 events, implying a distribution of stress drop with a power-law like behavior. In contrast, the bubble model of Durian, which is also applicable to a wet foam, predicts power-law distributions with an exponential cutoff [9, 10]. This model includes viscous dissipation between bubbles. Further work on this model demonstrated that the exponential cutoff increases as one approaches the 
melting point 14. However, the dependence on area fraction is weak, and true power-law behavior was never observed, even extremely close to the melting point [14]. Finally, simulations of a q-potts model of a sheared foam [13 did not assume a particular form for the dissipation. This work suggests that the distribution of topological rearrangements is not power-law like; however, the distribution of energy drops may be consistent with power-law behavior 13.

In order to distinguish between these various theoretical models, there has been limited experimental work on two-dimensional model foams [15, 16 and threedimensional foams [17. The work in Ref. [17] made indirect measurements of the bubble rearrangements and concluded that only local rearrangements occurred during flow. The work in Ref. [15] used Langmuir monolayers to form two-dimensional foam. This work directly measured the distribution of $\mathrm{T} 1$ events and concluded that the size distribution was not consistent with a powerlaw. In contrast, the work in Ref. [16] used a single layer of bubbles trapped between a glass plate and a water surface. This work focused on the bubbles whose topological class had changed. These measurements suggest that very large events can occur.

The apparent discrepancies in these measurements may not be as severe as it first appears. Detailed simulations of various models show that the size of T1 events and the number of bubbles involved in a rearrangement are not necessarily correlated [13,14]. Also, it is possible that the number of $\mathrm{T} 1$ events is not correlated with the size of a stress drop [13]. This is reasonable when one considers that not all T1 events will relieve an equivalent amount of stress. Therefore, to address the question of the scaling of the size distribution, it is critical to have direct measurements of the stress or energy drops. We have accomplished this for the case of another model, two-dimensional foam: a bubble raft 18,19.

A bubble raft consists of a single layer of bubbles placed on the surface of water. Bubble rafts have been used to model the flow behavior of amorphous solids [18,19. The bubble rafts are an ideal system for the study of two-dimensional foams for a number of reasons. They allow for both direct measurements of the macroscopic properties of the foam and the bubble dynamics. There are no confining glass plates, which can add complications as the shear rate is increased. Finally, one can control both the degree of order in the foam (by varying the distribution of bubble sizes) and the density of the foam with great precision. In this paper, we will report on the flow behavior of a disordered bubble raft with $\phi \approx 0.9$.

We generate flow of the bubble raft using a twodimensional Couette viscometer that is described in detail in Ref. 20]. The apparatus consists of two concentric cylinders oriented vertically. Water is placed between the cylinders, and the upper surface of the water is free. The outer cylinder consists of 12 individual pieces, so it can be expanded and compressed to adjust the density of the bubble raft. The working radius of the outer cylinder was $7.43 \mathrm{~cm}$. The inner cylinder is two pieces: a solid cylinder placed in the water and a second piece that is in contact with the bubble raft and fits over the solid cylinder. The second piece is hung by a torsion wire and is used to measure the stress, $\sigma$, on the inner rotor due to the bubble raft. It is important to note that this is a "two-dimensional stress" given by the force on the inner rotor in the tangential direction divided by the circumference of the rotor. Therefore, in terms of the torque, $\tau$ on the rotor and the radius of the rotor $r$, the stress is given by $\sigma=\tau /\left(2 \pi r^{2}\right)$. The radius of the inner cylinder was $3.84 \mathrm{~cm}$. A constant rate of strain is applied to the system by rotating the outer cylinder at a constant angular speed in the range $0.0005 \mathrm{rad} / \mathrm{s}$ to $0.01 \mathrm{rad} / \mathrm{s}$

The stress on the inner cylinder is determined from the angular displacement of the torsion wire supporting the inner cylinder. The angular displacement was measured using magnetic flux. A coil was attached to the torsion wire and suspended within a high-frequency magnetic field. The induced voltage was used to determine the angle of the coil. Typical values of the angle during shear ranged from $22^{\circ}$ to $40^{\circ}$, corresponding to stresses in the range of $4-23 \mathrm{dyne} / \mathrm{cm}$. The resolution in stress was set by our resolution in angular measurement and the torsion constant, $\kappa=570$ dyne $\mathrm{cm} / \mathrm{rad}$. The voltage signal was digitized using a 12-bit A to D converter in the computer. As a test of the noise level in the signal, the stress was monitored as a function of time without shear. In the absence of shear, the noise in the measured stress signal was at the level of the lowest bit in digitized signal, corresponding to changes in the stress of \pm 0.026 dyne $/ \mathrm{cm}$. Therefore, when measuring the changes in the stress, changes of \pm 0.026 dyne $/ \mathrm{cm}$ were ignored, providing a lower limit on the size of the stress drops.

The bubble raft is generated by flowing nitrogen through a solution of $44 \%$ by weight glycerine, $28 \%$ by weight water and $28 \%$ by weight Miracle Bubbles (Imperial Toy Corp.). The bubble size is fixed by the pressure and needle diameter. For the experiments reported on here, three different size bubbles ( $2 \mathrm{~mm}, 3 \mathrm{~mm}, 5$ $\mathrm{mm}$ ) were used, with approximately 500 bubbles of each size. The experiments were carried out over two hours. The coarsening of the foam without shear was monitored during this time, and no significant coarsening or stress drops due to coarsening were observed. However, after two-hours, the stability of the foam deteriorated rapidly, and shear resulted in severe rupture of bubbles.

Figure 1 is an image of a section of the bubble raft between the two cylinders. The bubble raft was monitored for slippage at both the outer and inner cylinder. For all of the experiments reported here, there was no slip between the first row of bubbles and the correspond- 
ing cylinder. Also, it was clear from the images that the stress drop events corresponded to rearrangements of the bubbles in the bulk of the foam. The average azimuthal velocity distribution was consistent with what one would expect for Couette flow. This is illustrated in Fig. 2 for an angular rotation of $0.01 \mathrm{~s}^{-1}$. The dashed line is the theoretical velocity profile for a Newtonian fluid, and the solid curve is the theoretical velocity profile for a shear-thinning fluid with a viscosity given by $\eta=m \dot{\gamma}^{n-1}$, where $m$ is a constant, $\dot{\gamma}$ is the shear rate, and $n=1 / 3$ [21]. It should be noted that the bubble model predicts shear-thinning with an exponent of approximately $1 / 3$ [22]. The fact that the velocity profile is consistent with the expected shear-thinning profile, and not the velocity profile of the water substrate, is strong evidence that the foam is acting independent of the water. Because the rate of strain varies as a function of radius, all reported values of for rates of strain are taken at the inner cylinder. Finally, it should be noted that this is very different behavior from that observed for two-dimensional foams confined between glass plates. In this case, exponential decay of the velocity profile was observed 23].

Figure 3 shows a typical response of the bubble raft to shear. The stress on the inner cylinder is shown as a function of the applied strain for a rate of strain of $3.1 \times 10^{-3} \mathrm{~s}^{-1}$. The two key features to notice are the initial elastic region where the stress increases linearly with strain and the subsequent region in which flow occurs. The intermittent nature of the flow is obvious in this plot. The yield strain was always of order 1 for all rates of strain.

Figure 4 shows the distribution of the stress drops for three different rates of strain. Here the size of the drop is normalized by the average stress per bubble. Also, as discussed above, any change in stress of \pm 0.026 dyne $/ \mathrm{cm}$ was ignored and does not enter into the plotted distribution. The distribution of stress drops is not consistent with a power-law for all length scales; however, for small stress drops, the distribution is consistent with a power law with an exponent of -0.8. For comparison, the exponent predicted for the bubble model is $-0.70 \pm 0.05$ [10]. Also, as predicted by the bubble model [14], the distribution is essentially independent of the rate of strain for small stress drops, and the large stress drop cutoff is weakly dependent on the rate of strain. This is difficult to see from Fig. 4, but is made clear by considering the average stress drop size. For example, for a rate of strain of $0.031 \mathrm{~s}^{-1}$, the average stress drop (normalized by the average stress per bubble) is 27.9 ; whereas, it is 47.0 for a rate of strain of $0.31 \mathrm{~s}^{-1}$.

We have conclusively demonstrated that the general features of the distribution of stress drops observed in the bubble raft are in agreement with the simulations reported in Ref. 10] and [14]. Specifically, the cutoff in the distribution for large stress drops is clear. Also, the viscosity of the bubble raft is consistent with a shear- thinning fluid with an exponent of $1 / 3$. Finally, the distribution of stress drops, particularly for the small stressdrops, is essentially independent of the rate of strain for the values studied here. This strongly suggest the existence of a quasi-static limit. Such a limit is particularly important for our geometry where the rate of strain varies across the system.

A number of important questions remain. First, what is the correspondence between the size of the stress drops and the number of bubbles that exchange neighbors? It is still possible that the larger stress drops are due to rearrangements involving a wide distribution of the number of bubbles, as reported in 13. Therefore, "systemwide" events are still a possibility. The current imaging system was not appropriate for careful tracking of bubbles throughout the system. Future improvements in the imaging of this system will enable this question to be addressed. Second, a detailed study of the flow behavior of bubble rafts as a function of both disorder (polydispertiy) and area fraction will provide further tests of the various two-dimensional models of foams. Of particular importance is the behavior as a function of area fraction to distinguish further between the predictions of the quasistatic simulations 11, 12] and the bubble model [10, 14.

The authors acknowledge funding from NSF grant CTS-0085751. M. Dennin acknowledges additional funding from the Research Corporation and Alfred P. Sloan Foundation. J. Lauridsen was supported by NSF Research Experience for Undergraduates grant PHY9988066 and a grant from UCI-UROP program. The authors thank Mingming Wu, Andrea Liu, and Doug Durian for useful conversations.

[1] There is a vast literature on foams and their flow behavior. Some reviews are: D. Weaire and S. Hutzler, The Physics of Foams, (Claredon Press, Oxford, 1999), J. Stavans, Rep. Prog. Phys. 56, 733 (1993), and A. M. Kraynik, Ann. Rev. Fluid Mech. 20, 325 (1988).

[2] M. E. Cates, J. P. Wittmer, J.-P. Bouchaud, and P. Claudin, Phys. Rev. Lett. 81, 1841 (1998).

[3] A. J. Liu and S. R. Nagel, Nature 396, 21 (1998).

[4] V. Trappe, V. Prasad, L. Cipelletti, P. N. Segre, D. A. Weitz, Nature 411, 772 (2001).

[5] Jamming and Rheology, eds. A. J. Liu and S. R. Nagel, (Taylor and Francis Group, 2001).

[6] K. Kawasaki, T. Nagai, and K. Nakashima, Phil. Mag. B 60, 399 (1989).

[7] K. Kawasaki, T. Okuzono, T. Kawakatsu, and T. Nagai, Proc. Int. Workshop of Physics of Pattern Formation ed. S. Kai (Singapore: World Scientific, 1992).

[8] T. Okuzono and K. Kawasaki, Phys. Rev. E 51, 1246 (1995).

[9] D. J. Durian, Phys. Rev. Lett. 75, 4780 (1995). 
[10] D. J. Durian, Phys. Rev. E 55, 1739 (1997).

[11] D. Weaire, F. Bolton, T. Herdtle, and H. Aref, Phil. Mag. Lett. 66, 293 (1992).

[12] S. Hutzler, D. Weaire, and F. Bolton, Phil. Mag. B 71, 277 (1995).

[13] Y. Jiang, P. J. Swart, A. Saxena, M. Asipauskas, and J. A. Glazier, Physical Review E 59, 5819 (1999).

[14] S. Tewari, D. Schiemann, D. J. Durian, C. M. Knobler, S. A. Langer, and A. J. Liu, Phys. Rev. E 60, 4385 (1999).

[15] M. Dennin and C. M. Knobler, Phys. Rev. Lett. 78, 2485 (1997).

[16] A. A. Kader and J. C. Earnshaw, Phys. Rev. Lett. 82, 2610 (1999).

[17] A. D. Gopal and D. J. Durian, Phys. Rev. Lett. 75, 2610 (1995).

[18] A. S. Argon and H. Y. Kuo, Mat. Sci. and Eng. 39, 101 (1979).

[19] D. Mazuyer, J. M. Georges, and B. Cambou, J. Phys. France 49, 1057 (1989).

[20] R. S. Ghaskadvi and M. Dennin, Rev. Sci. Instr. 69, 3568 (1998).

[21] R. B. Bird, R. C. Armstrong, and O. Hassuage, Dynamics of Polymer Liquids (Wiley, Newyork, 1977).

[22] S. A. Langer and A. J. Liu, Europhys. Lett. 49, 68 (2000).

[23] G. Debrégeas, H. Tabuteau, and J. -M. di Meglio, Phys. Rev. Lett. 87, 178305 (2001).

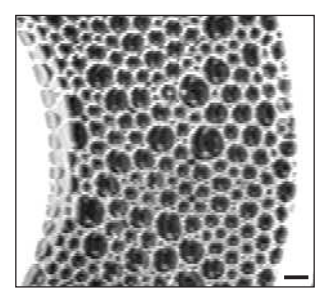

FIG. 1. This is an image of one section of a typical bubble raft. Part of both the inner and outer cylinder are visible. The black scale-bar in the lower right corner is $3.6 \mathrm{~mm}$.

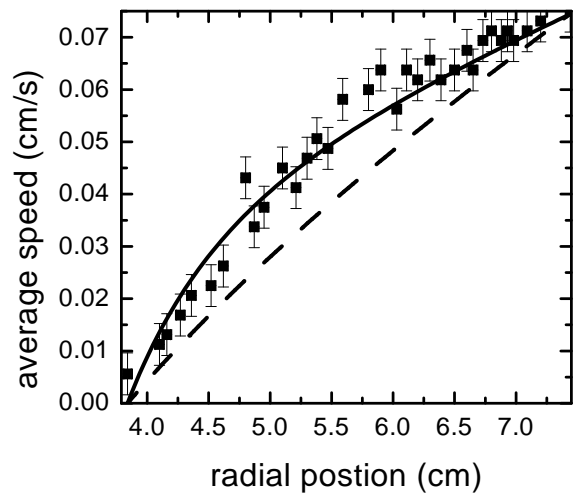

FIG. 2. Plot of average azimuthal speed of the bubbles versus radial position (solid symbols). The solid line is the theoretical velocity profile for a shear-thinning fluid with $\mathrm{n}$ $=1 / 3$ (see the text for details), and the dashed line is the theoretical profile for a Newtonian fluid.

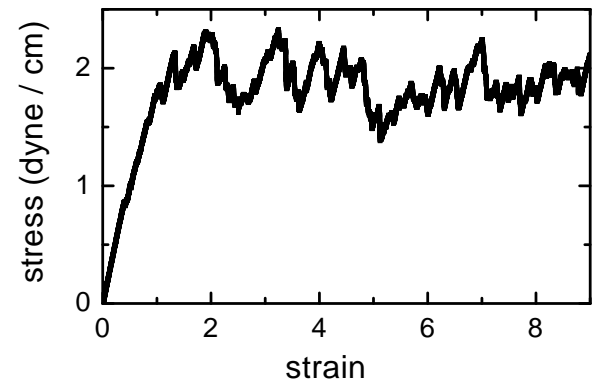

FIG. 3. Plot of the stress versus strain for a rate of strain of $3.1 \times 10^{-3} \mathrm{~s}^{-1}$.

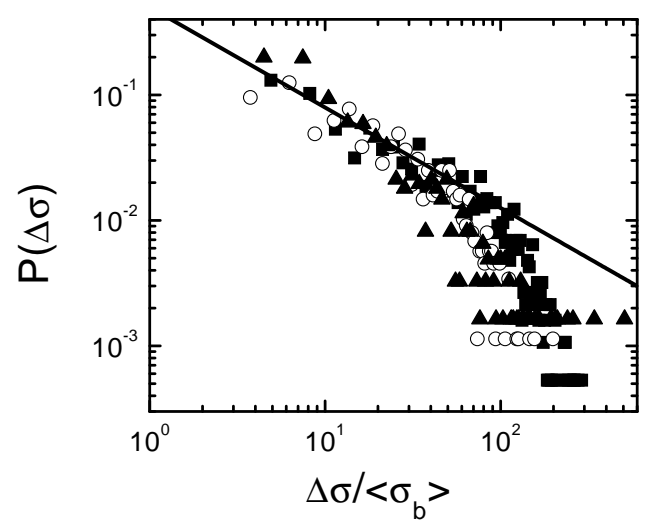

FIG. 4. Distribution of stress drops for three different rates of strain. Solid triangles are a rate of strain of $0.031 \mathrm{~s}^{-1}$, solid squares are a rate of strain of $0.31 \mathrm{~s}^{-1}$, and open circles are for a rate of strain of $0.48 \mathrm{~s}^{-1}$. The solid line has a slope of -0.8 and is a guide to the eye. 